\title{
Estimation of Properties of Liquid-Vapor Mixture of Some Refrigerants at High Pressure for Solar- Photovoltaic Refrigeration
}

\author{
A. Abdallah El Hadj ${ }^{1,2}$, M. Laidi ${ }^{1,2}$ C. Si-Moussa ${ }^{2}$ and S. Hanini ${ }^{2}$ \\ ${ }^{1}$ Department of chemistry, University \\ of Blida, USDB-1(Algeria). \\ ${ }^{2}$ LBMPT, University of Medea- LBPT- (Algeria) \\ E-mail: a_abdallahelhadj@univ-blida.dz
}

\begin{abstract}
In this work, a hybrid method based on neural network and particle swarm optimization is applied to literature data to develop and validate a model that can predict with precision vapor-liquid equilibrium data for the binary systems (hexafluoroethane (R116(1)), 1,1,1,2-tetrafluoroethane (R134a) and R1234ze). ANN was used for modelling the non-linear process. The PSO was used for two purposes: replacing the standard back propagation in training the ANN and optimizing the process. The training and validation strategy has been focused on the use of a validation agreement vector, determined from linear regression analysis of the predicted versus experimental outputs, as an indication of the predictive ability of the neural network model. Statistical analysis of the predictability of the optimized neural network model shows excellent agreement with experimental data (coefficient of correlation equal to 0.998). Furthermore, the comparison in terms of average relative deviation (AARD\%) between, the predicted results for the whole temperature and pressure range shows that the ANN- PSO model can predict far better the mixture properties than cubic equations of state.
\end{abstract}

\section{Key words}

R134a, Artificial neural networks, Refrigerant, Particle Swarm Optimization, Solar-Photovoltaic, Refrigeration.

\section{Introduction}

An increasing level of interest in solar- photovoltaic refrigeration system is recorded in the last decade due to its importance in many processes in particular the use of refrigerant mixtures to have renewable energy process with more efficiency and without constraints of pollution.

Modeling of such mixtures for the purpose of correlation and /or prediction is very important for simulation and analysis.

Artificial neural networks (ANNs) are computer based systems that are designed to simulate the learning process of neurons in the human brain. ANNs have been attracting great interest during the last decade as predictive models and pattern recognition. Artificial neural networks possess the ability to "learn" from a set of experimental data (e.g. processing conditions and corresponding responses) without actual knowledge of the physical and chemical laws that govern the system. Therefore, ANNs application in data treatment is especially important where systems present nonlinearities and complex behavior [1-6]. Artificial neural networks are a promising alternative modeling technique because it considers as an alternative tool for the classical model that can extract the relation from highly non-linear problems.

Particle swarm optimization (PSO) and ant colony optimization (ACO) are the most efficient algorithms compared with GA and the other methods. The choice of PSO technique (mainly for training the ANN) is justified by the remarkable optimization ability (the PSO has no overlapping and mutation calculation like genetic algorithm, and during the development of several generations, only the most optimist particle can transmit information onto the other particles, and the speed of the researching is very fast.

In this work, hybrid method based on neural network and particle swarm optimization (PSO) is applied to literature data to develop and validate a model that can predict with precision vapor-liquid equilibrium data for the binary system of hexafluoroethane (R116 (1)) ; 1,1,1,2tetrafluoroethane (R134a), R1234ze and R22). ANN was used for modeling the non-linear process. The PSO was used for two purposes: replacing the standard back propagation in training the ANN and optimizing the process. The training and validation strategy has been focused on the use of a validation agreement vector, determined from linear regression analysis of the 
predicted versus experimental outputs, as an indication of the predictive ability of the neural network.

\section{Modeling Using ANN And PSO}

Figure 1 gives a vapour-liquid equilibria inside the simple refrigeration process. The knowledge of composition of this mixture is of paramount interest in order to determine the operating conditions for high efficiency.

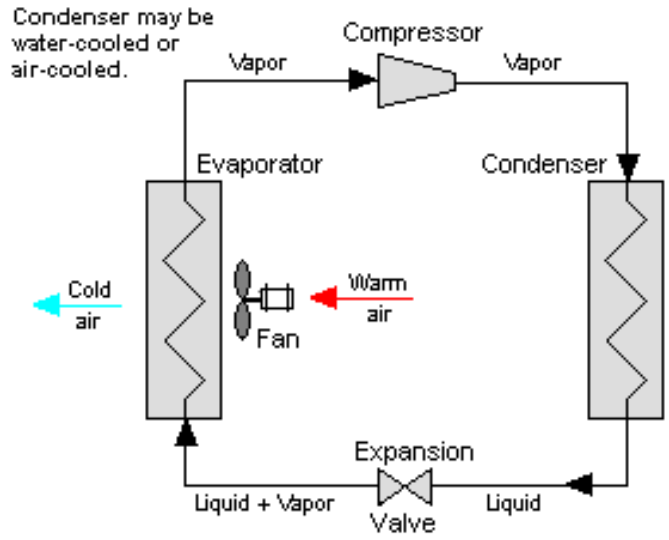

Figure1: Simple vapour compression refrigeration

In order to describe the phase behavior binary systems of hexafluoroethane (R116 (1)) and 1,1,1,2tetrafluoroethane (R134a) and R1234ze) by one model a total of six variable have been selected in this work. four have been selected as inputs variables; (The equilibrium temperature $(\mathrm{T})$, the critical temperature $(\mathrm{Tc})$, liquid fraction (xi) and critical pressure (Pc), and equilibrium pressure (P)) and vapour fraction (y2) as the output variable (Fig.1). A detailed description of the strategy used for the application of ANN modelling of phase equilibria has been described in a previous work.

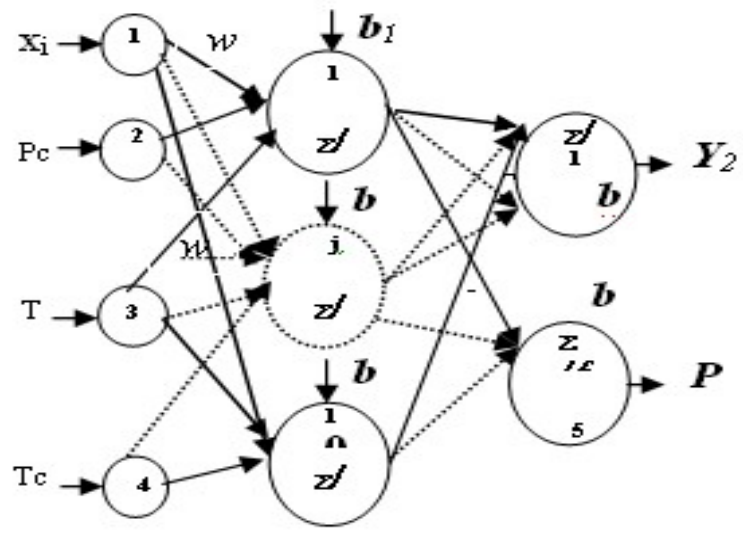

Fig. 2. Multi-layer feedforward neural network for the prediction of the refrigerants mixture properties.

The application of ANN modelling was performed using MATLAB ${ }^{\circ}$ (version 2014)

and the strategy proposed by Plumb and his coworkers [7-8] as follows:

1. The experimental data should be divided into a training set, a test set (when attenuated training is adopted) and a validation set. Each data set should be well distributed throughout the model space.

2. Initially, the model should be trained using the default training algorithm and network architecture. The parameters of the equation of the best fit (the slope and the $y$ intercept of the linear regression) or the goodness of fit (correlation coefficient, R) are determined for validation plots of the predicted versus the experimental properties of the validation data set. These parameters are used as a measure of the predictive ability of the model. Where the agreement vector values approach the ideal, i.e. $[\alpha=1$ (slope), $\beta=0$ (y intercept), $\mathrm{R} 2=1$ ], little improvement in predictive ability is to be expected. The ANN model with the best agreement vector is retained and the procedure is stopped

3 . Where the values of the parameters of the agreement vector vary greatly from the Ideal and the model is poorly predictive, modification of the number of hidden layer neurons is then considered.

4. If model performance remains unsatisfactory a systematic investigation of the effect of varying both the training algorithm and network architecture is required.

All the input and output data were scaled so as to have a normal distribution with zero mean and unit standard deviation using the following scaling equation:

$$
\text { Scaled value }=(\text { Actual value }-\mu) / \sigma
$$

Where $\mu$ and $\sigma$ : are the mean and standard deviations of the actual data respectively. The values of $\mu$ and $\sigma$ for the input and output data, referred to in Tables 1-3, are listed in Table 4.

MATLAB neural network toolbox contains various pre and post data processing methods.

When using the above scaling method, scaling and descaling are carried out by prestd and poststd MATLAB functions respectively.

The strategy of calculation is based on the variation of the topology of ANN model starting with default feedforward backpropagation NN type (newff MATLAB function), number of hidden layers and the number of neurons in each hidden layer. The optimization of ANN model parameters are performed using particle swarm optimization algorithm to minimize the objective function (OF), which is average absolute relative deviation (AARD \%) defined usually as:

$$
O F=A A R D(\%)=\frac{100}{N} \sum_{1}^{n} \frac{\left|y_{\text {calc }}-y_{\exp }\right|}{y_{\exp }}
$$

The structure of the optimized ANN model with number of neurons in input and output layers and hidden layers with activation functions and training algorithm used are shown in table I.

Table I - Structure of the optimized ANN model 


\begin{tabular}{|l|c|l|}
\hline Network type & 'Feedforwaed backpropagation': newff \\
\hline Layers & Number of neurons & Activation function \\
\hline Input layer & 4 & ------ \\
\hline $1^{\text {st }}$ Hidden layer & 6 & LogarithmicSigmoid: logsig \\
\hline Output layer & 2 & Linear: purelin \\
\hline Training Algorithm & 'Particle Swarm Optimization' : trainpso \\
\hline
\end{tabular}

\section{Results and Discussion}

In this work, the ANN modeling was carried out using MATLAB. The first global comparison between the solubility of solid drugs estimated by the ANN model versus experimental is shown through validation agreement plots. Based on the coefficient of correlation and the distribution data on $\mathrm{y}=\mathrm{x}$ line (figure 1). We can say that the ANN model gives a good agreement with experimental data for all treated systems.

Figures 4-5 show a comparison between experimental composition of two refrigerants and that estimated by RNSO-PSO. The figures show excellent agreement between experimental literature data (shown as white face markers) and the ANN predicted solubility (shown as fully face markers)

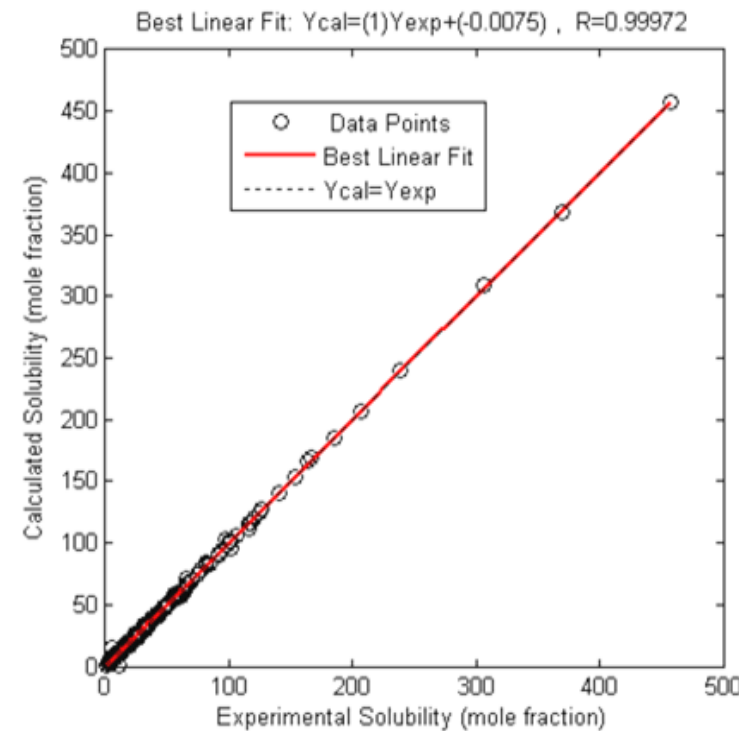

Fig. 3. Validation agreement plot of the most predictive model of the liquid mole fraction.

Another type of comparison is carried out using a comparison with relative deviation calculated between experimental data and the calculated pressure and composition with optimized neural network ANN-PSO (table 2)

Table 1: comparison in terms of AARD between between experimental equilibrium properties and those calculated with ANN-PSO

\begin{tabular}{|l|c|c|c|c|}
\hline Component & \multicolumn{2}{|c|}{ AARD (\%) } & \multicolumn{2}{c|}{ AARD (\%) } \\
\hline R116 & $\mathrm{X}_{\mathrm{i}}$ & $\mathrm{P}$ & $\mathrm{X}_{\mathrm{i}}$ & $\mathrm{P}$ \\
\hline $\mathrm{R} 134 \mathrm{a}$ & 1.1 & 0.05 & 0.92 & 0.12 \\
\hline
\end{tabular}

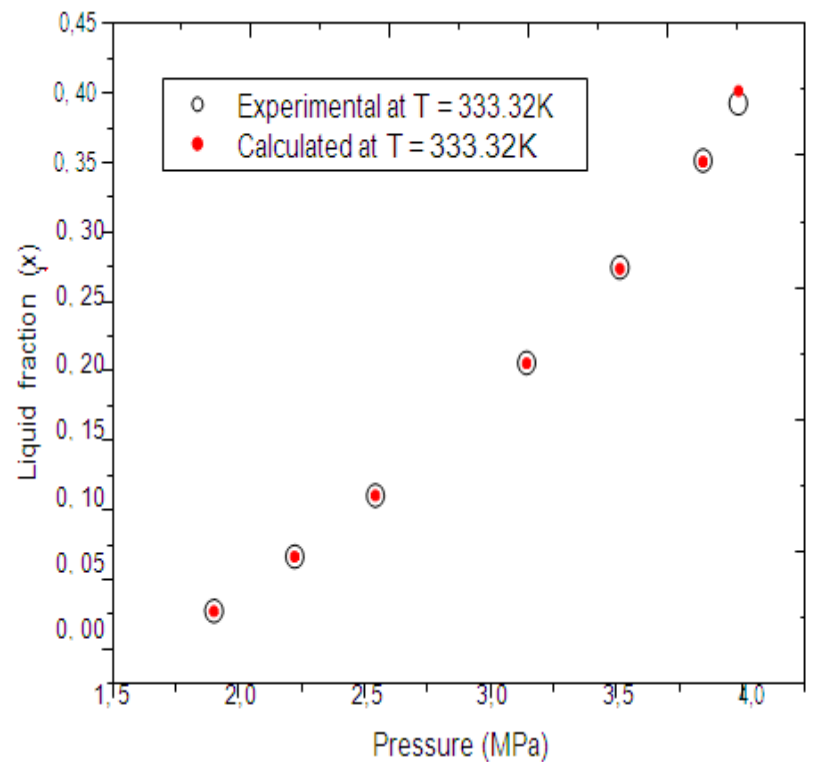

Fig. 4. Comparison between experimental composition of two refrigerants [9] and that estimated by RNSO-PSO at T $=333.32 \mathrm{~K}$

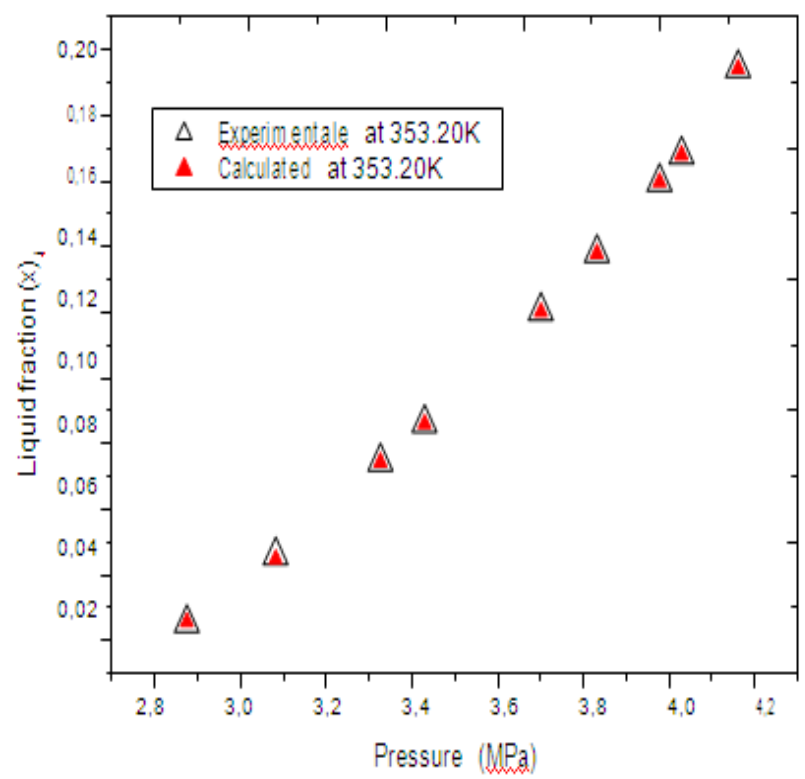

Fig. 5. Comparaison between experimental composition of two refrigerants [10] and that estimated by RNS-PSO at T= $353.20 \mathrm{~K}$ respectively 


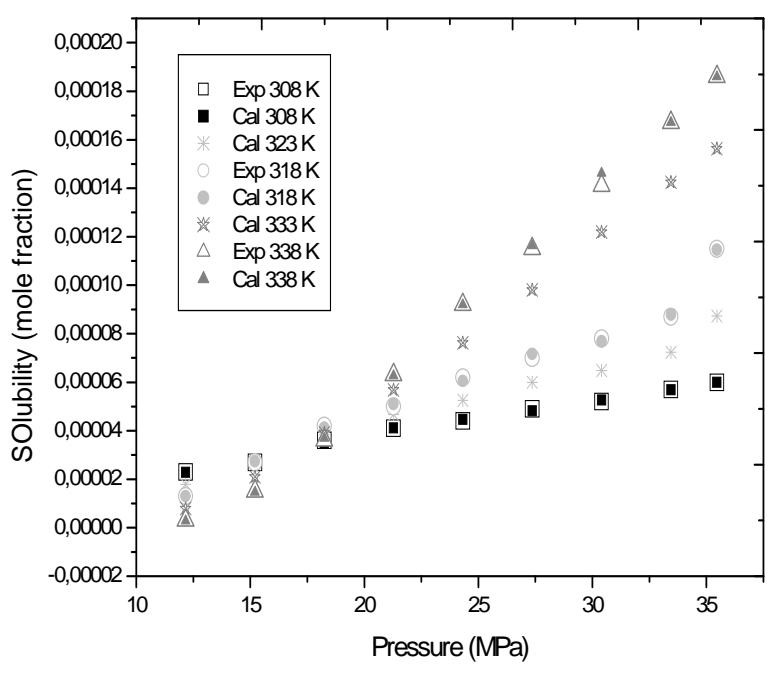

Fig. 6. Experimental composition of R116 that predicted by ANN model at various temperatures

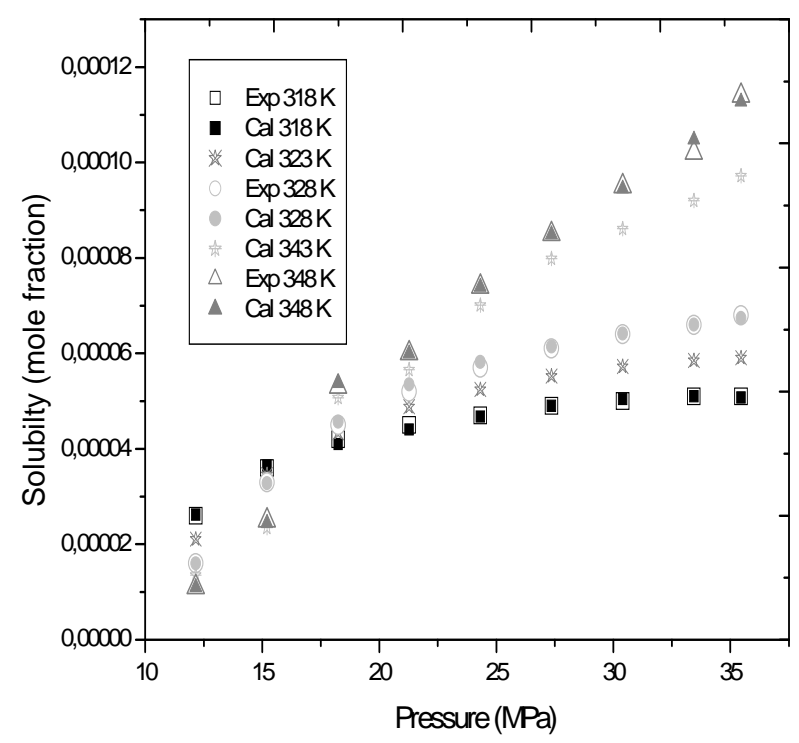

Fig. 7. Experimental composition of R1234ze and that predicted by ANN model at various temperaturas.

\section{Conclusion}

In this work, a hybrid method based on a feed forward artificial neural network and particle swarm optimization algorithm (PSO) has been used for developing model to predict is applied to literature data to develop and validate a model that can predict with precision vapor-liquid equilibrium data for the binary system of hexafluoroethane (R116 (1)); 1,1,1,2tetrafluoroethane (R134a) and R1234ze [11]) given the equilibrium temperature, the critical temperature, the critical pressure, acentric factor and the liquid mole fraction of the refrigerant. The optimized ANN consists of four neurons in the input later, one hidden layers with 17 neurons and two neurons in the output layer. This was obtained by applying a strategy based on assessing the parameters of the best fit of the validation agreement plots (slope and y intercept of the equation of the best linear fit and the correlation coefficient R) for the validation data set as a measure of the predictive ability of model. The comparison in terms of the AARD (\%) shows that the ANN model correlates the binary system properties with more precision, and PSO algorithm integrated to perform the ANN parameters has successfully given a predictive model with average absolute deviation that did not exceed $0.95 \%$.

\section{References}

[1] J. Bourquin, H. Schmidli, and P. van Hoogvest, H. Leuenberger, "Basic concept of artificial neural networks (ANN) modelling in the application of pharmaceutical development“, Pharm. Dev. Technol. 2, (1997), p.95.

[2] D.R. Baughman, and Y.A. Liu, "Neural Networks in BioProcessing and Chemical Engineering", Academic Press, New York (1995)

[3] R.B. Boozarjomehry, F. Abdolahi and M.A. Moosavian, "Characterization of basic properties for pure substances and petroleum fractions by neural network“" Fluid Phase Equilib., 231, (2005), p.188

[4] T. Khayamian and M. Esteki, "Prediction of solubility for polycyclic aromatic hydrocarbons in supercritical carbon dioxide using wavelet neural networks in quantitative structure property relationship“, J. Supercritical Fluids, 32, (2004), p.73.

[5] M. Lashkarbolooki, B. Vaferi, A. Shariati and Z. Hezave, "Investigating vapor-liquid equilibria of binary mixtures containing supercritical or near-critical carbon dioxide and a cyclic compound using cascade neural network“, Fluid Phase Equilibria, 343, (2013), pp. 24-29.

[6] M. Lashkarbolook, Z. S. Shafipour and Z. Hezave, "Trainable cascade-forward back- propagation network modeling of spearmint oil extraction in a packed bed using SCCO2“, J. of Supercritical Fluids, 73, (2013b), pp. 108-115.

[7] A. P. Plumb, R. C. Rowe, P. York and M. Brown, "Optimization of the predictive ability of artificial neural network (ANN) models: A comparison of three ANN programs and four classes of training algorithm“, Eur. J. Pharm. Sci., 25 (2005) pp. 395-405.

[8] P.R. Patnaik, "Applications of neural networks to recovery of biological products", Biotechnology Advances, 17, (1999), p. 477.

[9] H. Madania., A. Valtz, C. Coquelet, A. H.Meniaia, D. Richon, "Vapor-liquid equilibrium data for the (hexafluoroethane+1,1,1,2-tetrafluoroethane) system at temperatures from 263 to $353 \mathrm{~K}$ and pressures up to $4.16 \mathrm{MPa}$ “, Fluid Phase Equilibria, 268 (2008), pp. 68-73.

[10] M-J. Lee, C-F. Kou, J.W. Cheng and H-M. Lin, "Vaporliquid equilibria for binary mixtures of carbon dioxide with 1 , 2-dimethoxybenzene, 2-methoxyphenol, or p-cresol at elevated pressures“, Fluid Phase Equilibria, 162, (1999), pp. 211-224.

[11] X. Wang, Y. Sun, K. Kang, "Experimental investigation for the solubility of R1234ze (E) in pentaerythritol tetrahexanoate and pentaerythritol tetraoctanoate", Fluid Phase Equilibria, 400, (2015), pp. 38-42. 